\title{
Multi-objective model for logistics distribution programming considering carbon emission and service level
}

\author{
Tong He \\ School of Traffic and Transportation,Lanzhou Jiaotong University,Lanzhou Gansu 730070,China \\ sunnytonger@126.com
}

Keywords:Logistics distribution network; carbon emissions; logistics service level; multi-objective; priority-based tabu search algorithm

Abstract.In order to seek the low-carbon environmental protection and high service level of logistics distribution, a multi-objective optimization model of logistics distribution network is proposed.Firstly,the multi-objective optimization model of logistics distribution network is established by using the multi-objective optimization theory, which takes the total network logistics cost as the minimum,the least carbon emissions and the maximization logistics service level as the goal.Secondly,the fuzzy programming method is used to transform the multi-objective programming model into a single objective programming model,and the tabu search algorithm based on priority is used to solve the model.And the model and the algorithm are verified by example.The results shows that the 10 solutions and 10 logistic distribution schemes are affected by the subjective preferences of the decision-makers by solving the mutli-objective optimization model.In addition, the total cost of the logistics of each scheme is inversely proportional to the level of service.A scientific and feasible logistics network planning method with low carbon and high customer satisfaction is obtained.

\section{Introduction}

Energy and environmental issues has become the focus of attention of countries around the world.Lu Fan.(2011) proposed that the logistics industry itself is one of the larger energy consumption industries and a large carbon emissions ${ }^{[1]}$.Moreover,the construction of logistics system has been raised from the strategic level of the enterprise to the strategic level of social infrastructure. So low-carbon logistics become a new hot spot in theoretical research at home and abroad.The rational planning of logistics distribution can not only effectively reduce logistics costs and improve logistics service level,but also further promote the coordination of production and consumption, to ensure the balanced development of logistics system.

Logistics distribution planning issues in the logistics service level and carbon emissions,the predecessors have the following research:In the aspect of logistics service level,Xifeng TANG,Haijun MAO,Xuhong LI.(2008) proposed that the logistics service level of the logistics distribution center from different angles,and then put forward the quantitative formula of the logistics service level.In terms of carbon emissions ${ }^{[2]}$.Palmer(2007) established a integrated optimization model for freight vehicles with time window ${ }^{[3]}$. This model set distance and carbon emissions as the goal,and analyzed the effect of speed on model optimization under different congestion conditions influences.Fagerholt(2010) applied the shortest path method to study the optimization problem of sea route with time window, and reduced the carbon emission by optimizing the transportation speed ${ }^{[4]}$. 
From the above study,we can see that the factors that influence the logistics service level of the logistics distribution center are not considered comprehensive enough.From the existing path optimization study of carbon emissions minimization, most of the studies focused on the impact of velocity changes on carbon emissions, with little consideration of the effects of load changes on carbon emissions.In this paper,a method of quantifying logistics service level is put forward,considering the influence of the existing cargo turnover capacity in the distribution center and product delivery time on customer satisfaction, and propose a method to quantify the logistics service level.Considering logistics cost,carbon emission and logistics service level,this paper uses the quantitative logistics service level and considers the effect of load change on carbon emission. And the shortest path problem is solved by fuzzy programming method and priority-based tabu search algorithm. The purpose is to reduce the carbon emissions and improve the logistics service level as well as customer satisfaction. That can be a new concept of effective convergence of the sustainable development of the logistics industry and energy-saving emission reduction as well as building a conservation-oriented society.

\section{Model Construction}

Problem description.In this paper,the problem of vehicle routing optimization with minimal total logistics cost,minimum carbon emissions and the maximum level of logistics service can be described as:The composition is $a$ production plants, $k$ candidate logistics distribution centers and $n$ customer demand points.Each logistics distribution center that responsible for $n$ customers distribution has several vehicles with each Load capacity is $w$, and customer demand is $g$. We need to find the vehicle routing paths that satisfy the minimum $\mathrm{CO}_{2}$ emission, minimum total logistics cost and maximum logistics service level,and use the least vehicle.

\section{Assumptions.}

(1) Only one product is produced in each production plant;

(2) The location of the logistics distribution center and each customer is known;

(3) The demand for each customer is fixed and must be met;

(4) Yuqing Wang,Maozeng Xu.(2012) proposed that the vehicle is a kind of model and must not surpass its load capacity ${ }^{[5]}$;

(5) Each customer can only and must visit once;

(6) The $\mathrm{CO}_{2}$ emission is proportional to the fuel consumption of the vehicle. The fuel consumption of the vehicle is related to the distance traveled by the vehicle and the cargo load,and with the change of cargo load,the fuel consumption per unit tonnage is also different;

(7) The production of factory $H_{a}$ product $a$ can be distributed to a number of logistics centers, and the demand of product $a$ from the customer demand point $U_{n}$ can choose a logistics center for distribution;

(8) Decision-makers select a number of logistics distribution centers from the $b$ candidate logistics distribution center to as the actual opening logistics center. 


\section{Parameter variables.}

Table 1. Parameter Variables

Parameter Variables:Meaning $r$ :Number of Factories

$b$ :Number of Candidate Logistics Distribution Centers

$l:$ Number of factory vehicles $m:$ Number of vehicles in logistics distribution center

$e:$ the number of customers

$t_{i j}:$ The amount of $\mathrm{CO} 2$ emissions per unit kilometer in the process from customer $i$ to customer $\mathrm{j}$

$w$ :Vehicle loading

$d_{a k}:$ The distance from the

factory to the logistics distribution center

$c_{a k}:$ The unit freight from the

factory to the logistics distribution center

$d_{k n}:$ The distance from the

logistics distribution center to the customer demand point $c_{k n}:$ The unit freight from the logistics distribution center to the customer demand point
Parameter Variables:Meaning

$t_{a k}$ :The $\mathrm{CO} 2$ emissions per unit kilometer from the factory to the logistics distribution center

$t_{k n}$ :The $\mathrm{CO} 2$ emissions per unit kilometer from the candidate logistics distribution center to the customer demand point $g_{i}$ :The i-th customer's demand

\section{$\gamma_{k}$ :The Variable Cost Coefficient of the kth Candidate}

\section{Distribution Center}

$x_{\max }$ :The total quantity of goods that the distribution center can

handle at the same time at most

$x_{\max x}:$ The kth candidate distribution center for the processing capacity of the goods; the total amount of goods that the distribution center can handle the products simultaneously.

$c_{f i x k}:$ The annual cost of fixed costs for each candidate logistics center $B_{k}$

$u_{a n}:$ The customer demand is the static demand.The demand of product a from point of demand in a specific time period, and each customer demand for the same product demand can only have a distribution center to provide services

$d_{i j}:$ The distance from the customer $i$ to customer $j$

$t_{n}$ :Each customer demand point on the product delivery time have a certain time limit. Within the time limit required by the customer, the shorter the delivery time of the distribution center,the higher the corresponding logistics service level $F_{k}\left(x_{k}\right)$ :Distribution center of the existing cargo turnover capacity-related logistics service level, which is the relationship of non-linear function from the distribution center $k$ for one-time processing of the volume of goods $x_{k}$

Model optimization.First, the minimum carbon emissions logistics network is established under the certain distribution conditions with the minimum carbon emissions. Directed graph $G=\{N, A\}, \mathrm{N}$ is the node set; $A$ is the arc set;Number of nodes $n=[N]$,Number of $\operatorname{arcs} m=[A], s$ represents the 
source node, $t$ represents the destination node.

The calculation of carbon emissions is divided into two parts: The carbon emissions of the first part is from the factory to the candidate logistics center, and the carbon emissions of the second part is from the logistics center to the customer demand point.The distance between the logistics center and the customer demand,the logistics distribution center is numbered 0 , and the customer in turn numbered $\mathrm{i}(\mathrm{i}=1,2, \ldots, \mathrm{n})$. Firstly,the calculation formula of carbon emission is analyzed.As can be seen from the assumption (6), $\mathrm{CO}_{2}$ emissions are proportional to vehicle fuel consumption, vehicle fuel consumption is related to vehicle driving distance and cargo load.Therefore,the linear regression analysis of the measured data can get the linear relationship between the load rate and unit kilometer $\mathrm{CO}_{2}$ emissions, so as to calculate the $\mathrm{CO}_{2}$ emissions per unit kilometer and the total $\mathrm{CO}_{2}$ emissions of $\mathrm{Z}$ :

$$
\left.Z=\sum_{a=0}^{r} \sum_{k=0}^{b} \sum_{h=1}^{l} d_{a k} y_{a k h} t_{a k}+\sum_{k=0}^{b} \sum_{n=0}^{e} \sum_{z=1}^{m} \sum_{i=0 j=0}^{n} \sum_{i=1}^{n} \sum_{k=1}^{m} d_{i j} y_{i j k} t_{i j}\right) t_{k n} y_{n z}
$$

According to the study ${ }^{[6]}$,the relationship between fuel consumption and carbon dioxide emissions and the load carrying capacity of an ordinary 8 tons city distribution vehicle is shown in table 2 .

Table 2. The Relationship between Load Rate and $\mathrm{CO}_{2}$ Emission

\begin{tabular}{cccc}
\hline Load rate $x$ & $\begin{array}{c}\text { Diesel fuel } \\
\text { consumption/ } \\
(\mathrm{L} / \mathrm{km})\end{array}$ & $\begin{array}{c}\mathrm{CO}_{2} \text { emission } \\
\text { coefficient }(\mathrm{kg} / \mathrm{L})\end{array}$ & $\begin{array}{c}\mathrm{CO}_{2} \text { emissions } \\
\text { corresponding to } \\
\text { load rate }(\mathrm{kg} / \mathrm{km})\end{array}$ \\
\hline 0 (no-load) & 0.1842 & 2.61 & 0.4807 \\
0.25 & 0.1973 & 2.61 & 0.5149 \\
0.5 & 0.2105 & 2.61 & 0.5494 \\
0.75 & 0.2236 & 2.61 & 0.5835 \\
$1.0($ full load $)$ & 0.2368 & 2.61 & 0.6180 \\
\hline
\end{tabular}

Through fitting the data of Table 2,the relationship between load rate and $\mathrm{CO}_{2}$ emission per unit kilometer is given as:

$$
t_{c c_{2}}=0.481+0.137 x^{[6]}
$$

Secondly, the level of logistics services is measured by considering the delivery time and cargo handling capacity.The cargo handling capacity of the logistics distribution center refers to the logistics distribution center can immediately deal with the goods.After the product is delivered from the production plant to the logistics distribution center when the customer sends an order request.In addition, due to the different size of the distribution center,the ability to deal with goods are not the same. When $x_{k \leq} x_{\text {max }}, F_{k}\left(x_{k}\right)=1$ said logistics distribution center can deal with customer demand in time. With the increase of $x_{k}$, when increasing to $x_{k}>x_{\max }$, the logistics distribution center can not deal with the customer demand in time,and the customer satisfaction is reduced and the 
corresponding logistics service level drops.

The relationship between the quantity of handled goods at one time $x_{k}$ by the distribution center and the level of logistics service $F_{k}\left(x_{k}\right)$ can be expressed as follows:

$$
F_{k}\left(x_{k}\right)=\left\{\begin{array}{l}
1, x_{k} \leq x_{\max } \\
1-\left(\frac{x_{k}-x_{\max }}{\alpha x_{\max }}\right)^{\beta}, x_{k}>x_{\max }
\end{array}\right.
$$

Where A and B are customer-defined constant coefficients.

When a number of logistics distribution centers serve multiple clients, the level of service related to the cargo turnaround capacity of the logistics distribution center in the system is:

$$
F_{\text {tooll }}=\frac{\sum_{k} F_{k}\left(x_{k}\right) x_{k}}{\sum_{k} x_{k}}
$$

The multi-objective logistics network optimization model is established with the goal of minimizing the total logistics cost,minimizing carbon emission and maximizing logistics service level:

$$
\begin{aligned}
& \min Y\left(\delta_{K}, \delta_{a k n}\right)=\sum_{k} c_{f u k} \delta_{k}+\sum_{a k n} \sum \sum\left(d_{a k}+d_{k n}\right) c_{a k n} u_{a n} \delta_{a k n}+\sum_{k} \gamma_{k}\left(\sum_{a n} u_{a n} \delta_{a k n}\right)^{p} \delta_{k}
\end{aligned}
$$

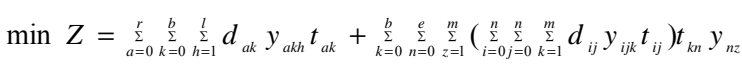

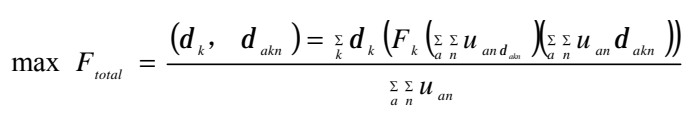

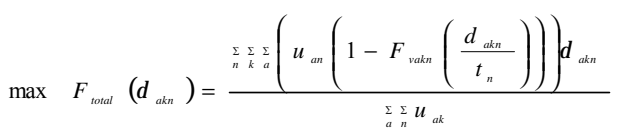

(8)

$$
\begin{aligned}
& \sum_{i=1}^{n} g_{i} x_{i k} \leq w(k=1,2, \ldots, m) \\
& \substack{m \\
k=1}_{k=1}^{m} x_{i k}=1(i=1,2, \ldots, n) \\
& \sum_{i=1}^{n} y_{j k}=x_{j k}(j=0,1, \ldots, n ; k=1,2, \ldots, k) \\
& \text { s.t. } \quad \sum_{i=0}^{n} y_{i j k}=x_{i k}(i=0,1, \ldots, n ; k=1,2, \ldots, m) \\
& \substack{n=1 \\
i=0 k=1}_{\substack{n \\
i=1}}^{n} y_{0 i k}=\sum_{j=0}^{n} \sum_{k=1}^{m} y_{j o k}(k=1,2, \ldots, m) \\
& y_{i j k}=0 \text { or } 1(i=0,1, \ldots, n ; j=0,1, \ldots, n ; k=1,2, \ldots, m) \\
& x_{i k}=0 \text { or } 1(i=0,1, \ldots, n ; k=1,2, \ldots, m) \\
& \delta_{k}=0 \text { or } 1(k=0, \ldots, b) \\
& \delta_{a k n}=0 \text { or } 1(a=0,1, \ldots, n ; k=1,2, \ldots, b ; n=1,2, \ldots, e) \\
& { }_{{ }_{k}} \delta_{a t n}=1, \forall a, n
\end{aligned}
$$

Where:Eq. 5 represents the minimum total cost of logistics; Eq. 6 represents the minimum carbon emissions;Eq. 7 represents the level of logistics service related to the existing capacity of the 
distribution center;Eq. 8 represents the satisfaction rate of multiple distribution centers for customer delivery time within their respective service areas;Eq. 9 is the constraint condition which respectively are the vehicle load constraints; each customer must have and only one vehicle for his services;only one vehicle arrives and leaves each customer (proposed by Christofides N,Eilon S.(1969). ${ }^{[7]}$ ); each vehicle starting from the logistics and distribution center,and finally back to the logistics and distribution center;parametric variables;Each demand point for the same product demand can only choose a logistics center to provide services.

\section{Design of search alogoritm based on priority.}

In the model,three goals are set,which belong to the NP problem. The neighborhood of the initial loop is constructed by the tabu search algorithm based on priority,and then the best individual is selected to get the local optimal solution.

Taboo search algorithm principle and implementation steps.Gendreau M.,Hertz A.,Laporte G.(1994).Proposed that an initial solution is generated as the current solution according to the stochastic method,when the tabu search algorithm is used to solve the combinatorial optimization problem $^{[8]}$. Then, the solution is searched in the neighborhood of the current solution, and the best solution is the new current solution.

We first get the shortest path scheme corresponding to the solution,and then judge whether the path satisfies the constraint condition of the problem and calculate the objective function value of the path scheme.Under the premise of satisfying the constraint condition of the problem, the better objective function value, the higher the quality of the solution. Set the neighbors: $+(v)=$ all sets of points that reach $\mathrm{v}\}, \mathrm{N}-(\mathrm{v})=\{$ all point sets starting from $\mathrm{v}\}$; The representation of the solution:give each vertex a priority (number), different points corresponding to different weights,point set:v1,v2, ..vn;Priority:P $(\mathrm{v} 1)=1, \mathrm{P}(\mathrm{v} 2)=2, \ldots, \mathrm{P}(\mathrm{vn})=$ n.Neighborhood search method:randomly select a number of pairs of vertices, exchange the weights between each vertex.The method is a neighborhood operation method which randomly selects two elements in the solution and exchange its values.For example.Let a solution $\mathrm{S}=123456789$, randomly generated exchange points for the first four and seven,then the implementation of the two exchange can get a neighbor of the original solution $\mathrm{A}=123756489$. The best solution of each iteration is put into the tabu list as a taboo object. The taboo length is a constant whose value is determined according to the size of the problem.A number of neighbors are randomly chosen as candidate sets from the neighborhood of the current solution. When the optimal object of the candidate set is better than the historical optimal solution,the object is forbidden.Termination criteria for iterating the specified number of steps:If the program reaches the preset number of iterations or the optimal solution is obtained continuously in a fixed period,the computation can be terminated by one of the two methods, in which the fixed period is set to 0.6 times of the iteration number of the algorithm.

When the tabu search algorithm is used to solve combinatorial optimization problems, the steps are as follows (taking the objective function minimization as an example):

Step 1:Select an initial solution $x^{\text {now }}$; let the tabu list with $H=\Phi$;

Step 2:If the termination criterion is satisfied,go to Step 4; otherwise,select the candidate set that satisfies the tabu requirement in $x^{\text {now }}$ 's neighborhood $N\left(X^{\text {now }}\right)$, and go to step 3;

Step 3:Choose the best solution $x^{\text {best }}$ in C-N (xnow), let $x^{\text {now }}=x^{\text {best }}$, then update the tabu list and go to step 2.

Step 4:Output the calculation result and stop.

Algorithm implementation.Xiao Li.et.al.(2006),concluded that the optimal tabu in the 
candidate set may be deleted during the searching process,so the candidate set does not have all the forbidden states ${ }^{[9]}$. The search is continued near the last initial solution,when the candidate solution set is empty. The time complexity of the algorithm is $o\left(n^{2}\right)$.

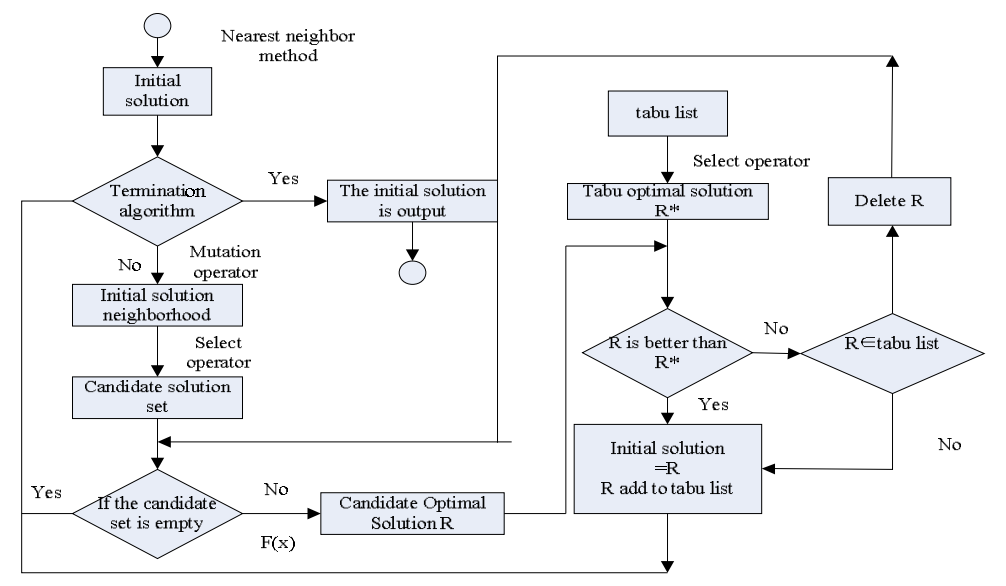

Fig. 1 Algorithm implementation process

Encoding:In order to improve the efficiency of the algorithm, this paper uses natural number coding.In the process of arriving at the logistics distribution center,a feasible route of the optimization problem can be programmed as length $b+l+1 .\left(0, k_{11}, k_{k 2}, \ldots, k_{k s}, 0, k_{21}, k_{22}, \ldots, k_{2 l}, 0, \ldots 0, k_{m l}, \ldots, k_{m v}, 0\right), k_{c j}$ represents the $c j$ customer.That can be understood as the first vehicle in the logistics distribution center to reach the customer demand point in the process, the optimization of a feasible route can be compiled for the length is $e+m+1 .\left(0, i_{11}, i_{i 2}, \ldots, i_{i s}, 0, i_{21}, i_{22}, \ldots, i_{2 l}, 0, \ldots 0, i_{m l}, \ldots, i_{m v}, 0\right), i_{k j}$ represents the $k j$ customer.That can be understood as the first car starting from the logistics distribution center 0,after the customer $i_{11}, i_{i 2}, \ldots, i_{i s}$ back to the logistics distribution center,forming a sub-path 1, the second car from the logistics and distribution center 0 ,after the customer $i_{21}, i_{22}, \ldots, i_{2 l}$ return to the logistics distribution center,forming a Path 2,and so forth,until all the customer's distribution requirements are met.

Decoding:Starting from the origin $(v=1)$, find the largest point $v^{\prime}$ of its weight in $\mathrm{N}-(\mathrm{v})$, and so on,until $v^{\prime}=n \quad(n$ is the end point).

\section{Example Analysis.}

Example selection.Model data:The model data is provided by a company in Lanzhou, which consists of 2 factories, 6 logistics distribution centers and 12 customer demand points. The cargo handling capacity of the logistics distribution center is shown in Table 3.The vehicle speed of transportation and distribution of products from the factory to the demand point is subject to mean of $50 \mathrm{~km} / \mathrm{h}$, the variance of 100 normal distribution, unit freight $c_{a k n}=5 \mathrm{yuan} / \mathrm{km}$, vehicle tonnage of 8 tons. The require is that reasonable arrangements for the route of the vehicle to minimize carbon emissions. 
Table 3. The relevant data of theLogistics and distribution center.

\begin{tabular}{ccccccc}
\hline Logistics distribution center & 1 & 2 & 3 & 4 & 5 & 6 \\
\hline Cargo handling capacity & 130 & 160 & 100 & 190 & 150 & 180 \\
Production plant $1(\mathrm{~km})$ & 120 & 110 & 135 & 80 & 140 & 100 \\
Production plant $2(\mathrm{~km})$ & 90 & 110 & 85 & 145 & 130 & 165 \\
Fixed cost $c_{\text {fuk }} / 10^{4}$ yuan & 1.2 & 1.4 & 0.8 & 1.7 & 1.5 & 1.7 \\
Variable cost coefficient $\delta_{k} /($ yuan $)$ & 9 & 8 & 7 & 6 & 8 & 9 \\
\hline
\end{tabular}

Table 4. The relevant data of the customer demand point. $(\mathrm{km})$

\begin{tabular}{ccccccccccccc}
\hline the customer demand point & 1 & 2 & 3 & 4 & 5 & 6 & 7 & 8 & 9 & 10 & 11 & 12 \\
\hline Logistics distribution center 1 & 155 & 187 & 136 & 128 & 200 & 160 & 135 & 80 & 56 & 97 & 120 & 28 \\
Logistics distribution center 2 & 135 & 110 & 185 & 179 & 156 & 86 & 189 & 230 & 190 & 114 & 156 & 150 \\
Logistics distribution center 3 & 165 & 220 & 145 & 199 & 300 & 179 & 260 & 135 & 99 & 72 & 114 & 84 \\
Logistics distribution center 4 & 200 & 170 & 136 & 75 & 165 & 155 & 180 & 315 & 45 & 89 & 110 & 79 \\
Logistics distribution center 5 & 310 & 260 & 165 & 360 & 135 & 230 & 310 & 179 & 180 & 160 & 140 & 150 \\
Logistics distribution center 6 & 180 & 150 & 155 & 270 & 135 & 150 & 125 & 188 & 170 & 150 & 130 & 165 \\
Demand for product 1 & 39 & 20 & 32 & 26 & 35 & 78 & 49 & 64 & 34 & 13 & 24 & 40 \\
Demand for product 2 & 26 & 54 & 39 & 49 & 24 & 24 & 31 & 45 & 15 & 45 & 8 & 18 \\
Delivery time & 4 & 5 & 4 & 3 & 6 & 5 & 3 & 7 & 2 & 7 & 4 & 5 \\
\hline
\end{tabular}

Solution.Firstly, the single objective programming problem of the total logistics cost,the carbon emission and the logistics service level in the logistics distribution network are solved separately,and the fitness of the reciprocal bit evaluation function of each objective function value is defined.Secondly,the optimal solution is reduced appropriately,and the ideal values of each single-objective programming problem are obtained by rounding.At the same time,the poor solution is amplified appropriately, and the ideal values of each single-objective programming problem are obtained by rounding. The calculation results are shown in Table 5.

Table 5. The ideal value and the worst value of logistics costs and carbon emissions

\begin{tabular}{cccc}
\hline $\begin{array}{c}\text { Each single-objective } \\
\text { planning problem }\end{array}$ & $f_{1} / 10^{6}$ yuan & $f_{2} / 10^{4} \mathrm{~km}$ & $f_{3} / \%$ \\
\hline The ideal value & 10 & 24 & 60 \\
The worst value & 16 & 70 & 95 \\
\hline
\end{tabular}

Note: $f_{1}$ is the total logistics costs; $f_{2}$ is carbon emissions; and $f_{3}$ is the logistics service level which is related to cargo turnover capacity and delivery time.

Then,according to the results of each single objective programming, the fuzzy programming method is used to transform the multi-objective into single-objective programming, and the tabu search algorithm based on priority is combined to solve.

The total fuzzy membership is defined as the fitness of the evaluation function,and the weight combinations of different fuzzy memberships are set up for the different schemes of running, then the computer simulation is carried out.On the $\mathrm{VC}++6.0$ platform, a tabu search algorithm based on priority is implemented to solve the problem of logistics distribution network and vehicle routing problem. The experiments are carried out on the computer whose main frequency is $133 \mathrm{MHz}$ and 
the memory is $16 \mathrm{MB}$. The number of iterations is $40 \mathrm{~N}$, the neighborhood size is $24 \mathrm{~N}$, the candidate set size is 10 , the tabu list size is $2 \mathrm{~N}$ ( $\mathrm{N}$ is the number of nodes), and the multi-objective optimization results can be obtained after decoding. The calculated results are shown in table 6 .

Table 6. Calculation results

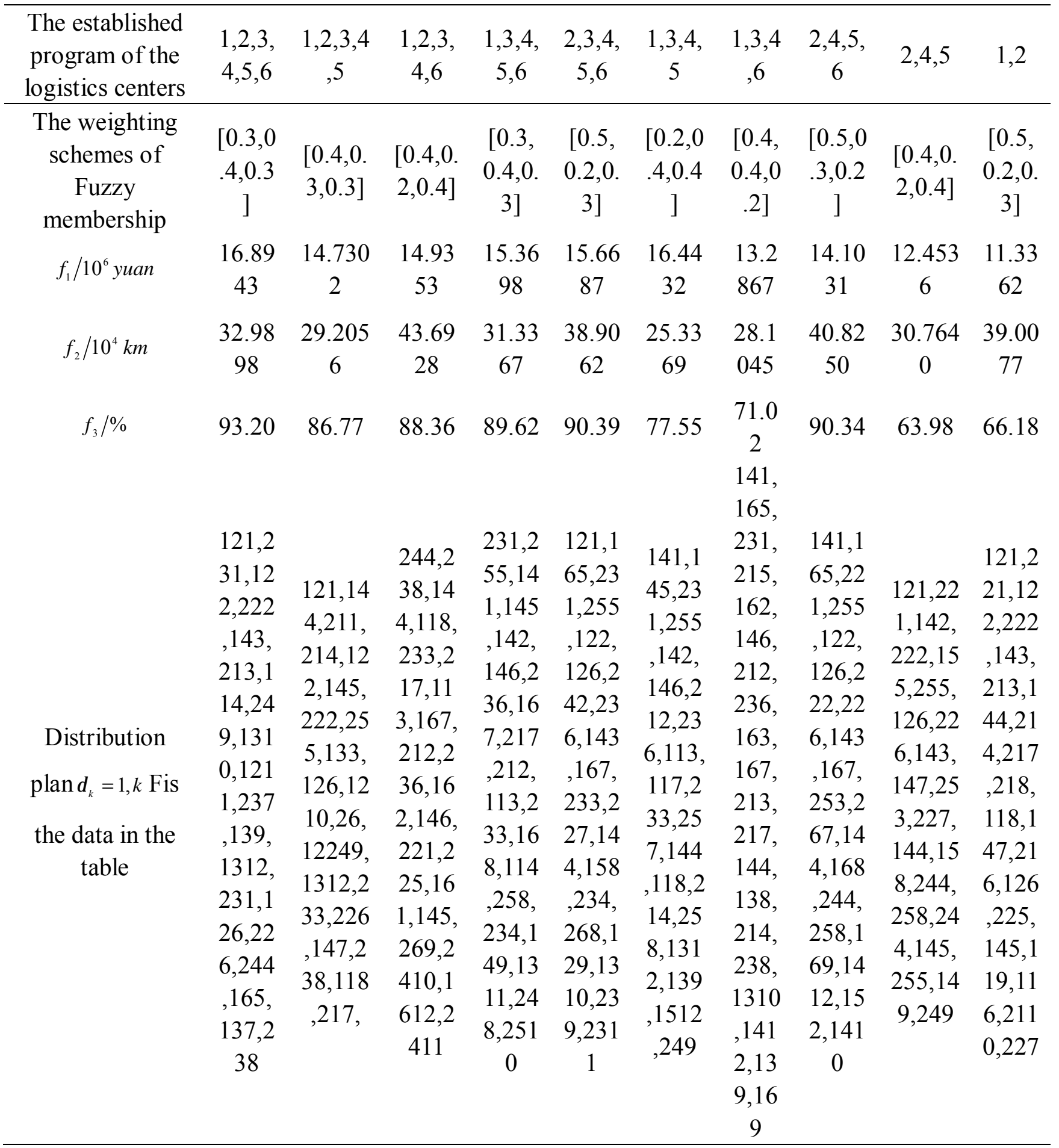

Note: $f_{1}$ is the total logistics costs; $f_{2}$ is carbon emissions; and $f_{3}$ is the logistics service level which is related to cargo turnover capacity and delivery time.

Multi-objective optimization problem can be summarized as a single objective: 


$$
\begin{aligned}
& \min f(x)=\left(f_{1}(X), f_{2}(X), \ldots, f_{L}(X)\right) \\
& \text { s.t. } G(X) \leq 0
\end{aligned}
$$

Where $\mathrm{A}$ is the sub-objective function, $\mathrm{B}$ is the decision variable, and $\mathrm{C}$ is the constraint.

Different weights are assigned to A,which can be transformed into a single-objective minimization problem for solving the linear weighted sums of each sub-goal.

$$
\min F(X)=\sum_{l=1}^{L} \lambda_{l} f_{l}(X)
$$

Where, $\lambda \in V=\left\{\lambda=\left(\lambda_{1}, \lambda_{2}, \ldots, \lambda_{L}\right) \mid \Sigma \lambda_{l}=1 \cup \lambda_{l} \geq 0\right\}$

The weight of each sub-object and the priority of each vertex are taken into account to optimize the logistics distribution network and vehicle routing ${ }^{[10]}$.

The vehicles running path from customer distribution to customer distribution is as follows:

Vehicle $1: 0 \rightarrow 10 \rightarrow 2 \rightarrow 5 \rightarrow 9 \rightarrow 6 \rightarrow 3 \rightarrow 7 \rightarrow 8 \rightarrow 11 \rightarrow 12 \rightarrow 1 \rightarrow 0$

Vehicle $2: 0 \rightarrow 5 \rightarrow 9 \rightarrow 3 \rightarrow 10 \rightarrow 4 \rightarrow 2 \rightarrow 11 \rightarrow 12 \rightarrow 8 \rightarrow 1 \rightarrow 0$

Vehicle $3: 0 \rightarrow 1 \rightarrow 6 \rightarrow 9 \rightarrow 8 \rightarrow 2 \rightarrow 7 \rightarrow 11 \rightarrow 5 \rightarrow 4 \rightarrow 12 \rightarrow 0$

Vehicle $4: 0 \rightarrow 2 \rightarrow 4 \rightarrow 11 \rightarrow 7 \rightarrow 6 \rightarrow 5 \rightarrow 3 \rightarrow 10 \rightarrow 12 \rightarrow 0$

Vehicle $5: 0 \rightarrow 12 \rightarrow 3 \rightarrow 7 \rightarrow 10 \rightarrow 4 \rightarrow 8 \rightarrow 6 \rightarrow 11 \rightarrow 9 \rightarrow 0$

Vehicle $6: 0 \rightarrow 8 \rightarrow 3 \rightarrow 4 \rightarrow 10 \rightarrow 11 \rightarrow 9 \rightarrow 12 \rightarrow 6 \rightarrow 5 \rightarrow 0$

From the calculated results, the greater the number of logistics distribution centers, the higher the logistics cost.But the level of logistics services is higher,which is related to the turnover capacity of goods and the delivery time.

While opening fewer logistics centers can save logistics costs, the service level is relatively low.Decision makers can choose appropriate decision solution in solution according to the business strategy,giving priority to cost,carbon emissions or service level.

Under different fuzzy membership weight, the logistics network is different,you can choose to open the logistics distribution center is different,the service object of each logistics distribution center is not the same.The denouement set is made with the multi-objective solution results under the three kinds of weight when the weight coefficient of the logistics cost is large,the corresponding carbon emissions are higher and the logistics service level is lower,which makes the total logistics cost of the network smaller. When the weight coefficient of carbon emissions is large,the total cost and logistics service level are relatively lower,the network carbon emissions is small. When the weight coefficient of logistics service level is large,the total cost and carbon emissions are correspondingly are higher,and the network logistics service level is higher.

\section{Conclusions}

In this paper,under the development environment of the logistics industry advocating energy saving and emission reduction,aiming at the problem of logistics distribution model,a multi-objective logistics network planning model is constructed with minimum total logistics cost,minimum carbon emission and maximum level of logistics service.Considering the impact of the change of load capacity on carbon emission and the factors of the turnover capacity of logistics distribution center and product delivery time, the quantitative calculation method of logistics service 
level is put forward.The multi-objective programming model is transformed into a single objective programming model by using fuzzy programming method.And the traditional tabu search algorithm is improved.In addition the weighting method of fuzzy membership degree and the tabu search algorithm based on priority are used to solve the model. A classical example is analyzed by using the model and algorithm, and good results are obtained.

(1)Compared with the tabu search algorithm based on the representation of directed edge permutation in the existing literature.In this paper,the tabu search algorithm based on priority has the advantages of natural representation of the solution,easy understanding of the algorithm,high computational efficiency and fast convergence speed.It is proved that the model and algorithm can solve this kind of network path problem well.

(2) This paper constructs the idea of tabu search algorithm of vehicle path and the new solution design and evaluation algorithm and other algorithm strategies in the algorithm, which has certain reference value to solve the similar combinatorial optimization problem.

However,each demand point discussed in this paper has a fixed demand for product demand.The further study is analysising the optimization model of logistics distribution location under stochastic demand,and the carbon emission problem when the vehicle speed and load are taken into account.

\section{Reference}

[1] Lu Fan.Research on Development Path of Low-carbon Logistics.China's Circulation Economy,25(8):46-51(2011).

[2] Xifeng TANG,Haijun MAO,Xuhong LI.Logistics facility location model based on reliability within the supply chain.Proceedings of the 4th IEEE International Conference on Management of Innovationand Technology.Bangkok:[s.n.],1099-1103(2008).

[3] Palmer.A.The development of an integrated routing and carbon dioxide emissions model for goods vehicles.Cranfield:Cranfield University,83-90(2007).

[4] Fagerholt K,Laporte G,Norstad I.Reducing fuel emissions by optimizing speed on shipping routes.Journal of the Operational Research Society,61(3):523-529(2010).

[5] Yuqing Wang,Maozeng Xu.Study on generalized TSP model based on minimum carbon emission.Mathematics in Practice Theory,42(8):69-75(2012).

[6] ZHU Changzheng, LI Yanling.Study on vehicle routing optimization problem based on carbon emissions minimizing.Computer Engineering and Applications,49 (22): 15-18(2013).

[7] Christofides N,Eilon S.An algorithm for the vehicle-dispatching problem.Operational Research Quarterly,20(3):309-318(1969).

[8] Gendreau M.,Hertz A.,Laporte G.A tabu search heuristics for the vehicle routing problem. Management Science,40:1276 1290(1994).

[9] Xiao Li,Guangyuan Li,Yi He.et.al.Structure Optimization of Fuzzy Neural Network Based on Tabu Search.Computer Science,33(7):217-219(2006).

[10]Jiefeng Xu,james P.K.A network flow-based tabu search heuristic for the vehicle routing problem. Transportation Science,30(4):379 393(1996). 